\title{
Fibrodysplasia Ossificans Progressiva in a 23-Year-Old Male
}

\author{
Ahmed-Al-Mustaque ${ }^{1}$, Ahmed Al Montasir ${ }^{2}$, Mashah Binte Amin ${ }^{3}$ \\ Received: August 14, 2013 Accepted: March 13, 2014
}

\begin{abstract}
Fibrodysplasia Ossificans Progressiva (FOP) is an inherited disease in which progressive ossification of striated muscles, tendons, ligaments and other connective tissues forming bridges of extra bones across the joints leads to severe disability and there are associated characteristic congenital skeletal malformations. FOP is also known as Stoneman's syndrome. The case we report here is a 23-year-old male with the clinical and radiologic characteristics of FOP.
\end{abstract}

Key words:Fibrodysplasia Ossificans Progressiva (FOP); Autosomal dominant; Bony exostosis

J Enam Med Col 2014; 4(2): 126-129

\section{Introduction}

Fibrodysplasia ossificans progressiva (FOP) is an autosomal dominant disorder. It is a rare disease, affecting one out of two million people worldwide. ${ }^{1}$ Eight hundred confirmed cases have so far been reported across the globe. ${ }^{2}$ Diagnosis of FOP can be made based on the patient's characteristic signs and symptoms of the disorder. ${ }^{1}$

\section{Case report}

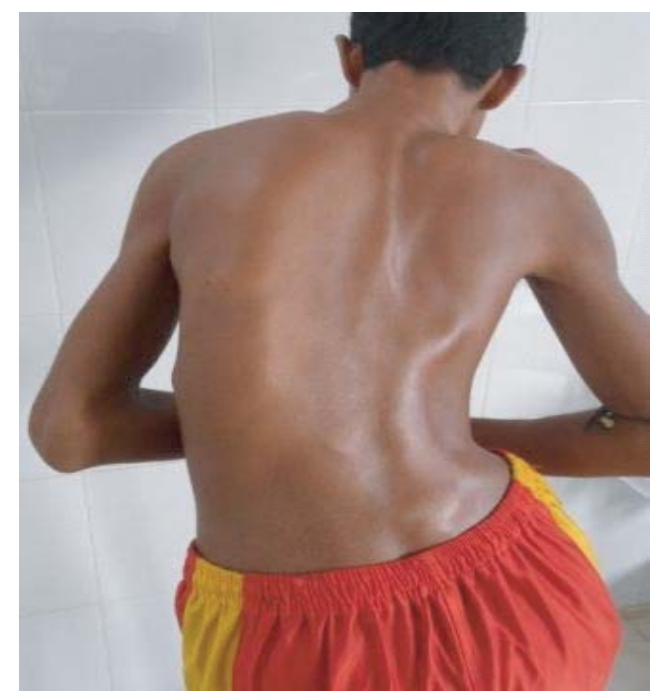

Fig 1. Scoliosis and bony exostosis in paravertebral region

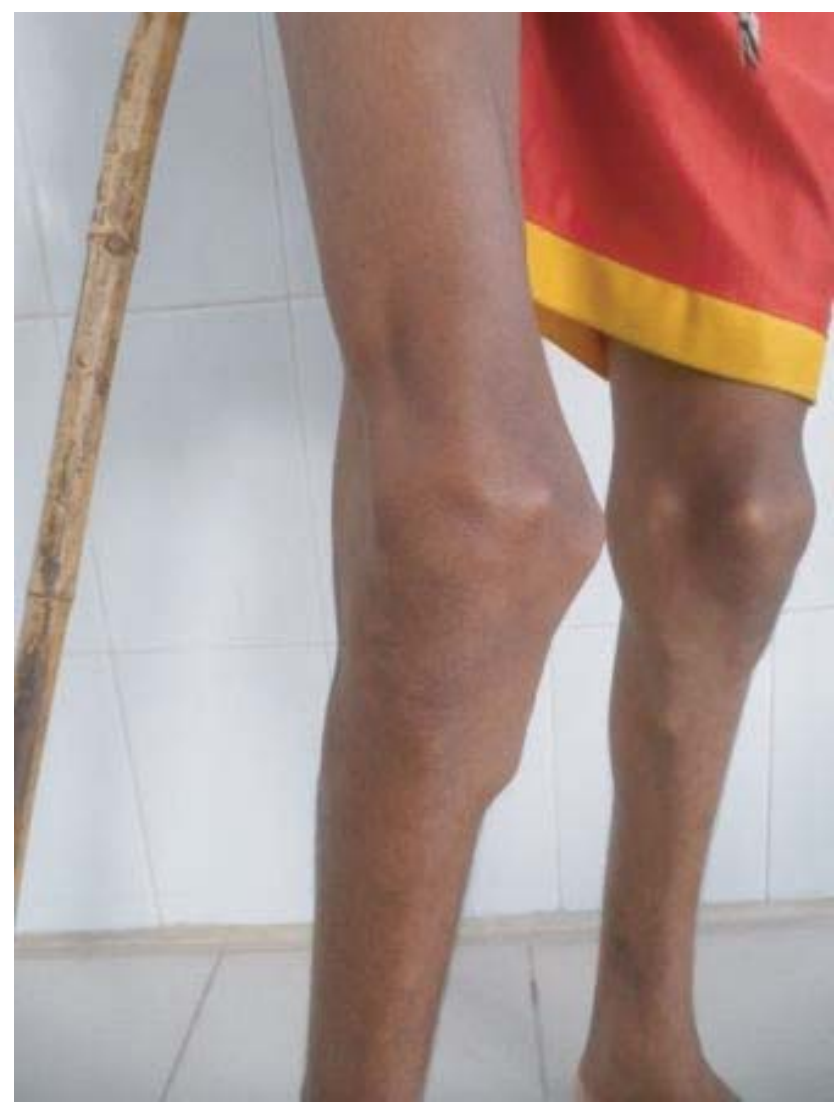

Fig 2. Bony exostosis around both knee joints

1. Assistant Professor, Department of Orthopedics, TMSS Medical College, Bogra

2. Resident Physician, Department of Medicine, Rafatullah Community Hospital, Bogra

3. Consultant, Department of Radiology \& Imaging, Abeda Memorial Hospital, Tongi, Dhaka

Correspondence Ahmed-Al-Mustaque,Email: mustaque_ortho@live.com 


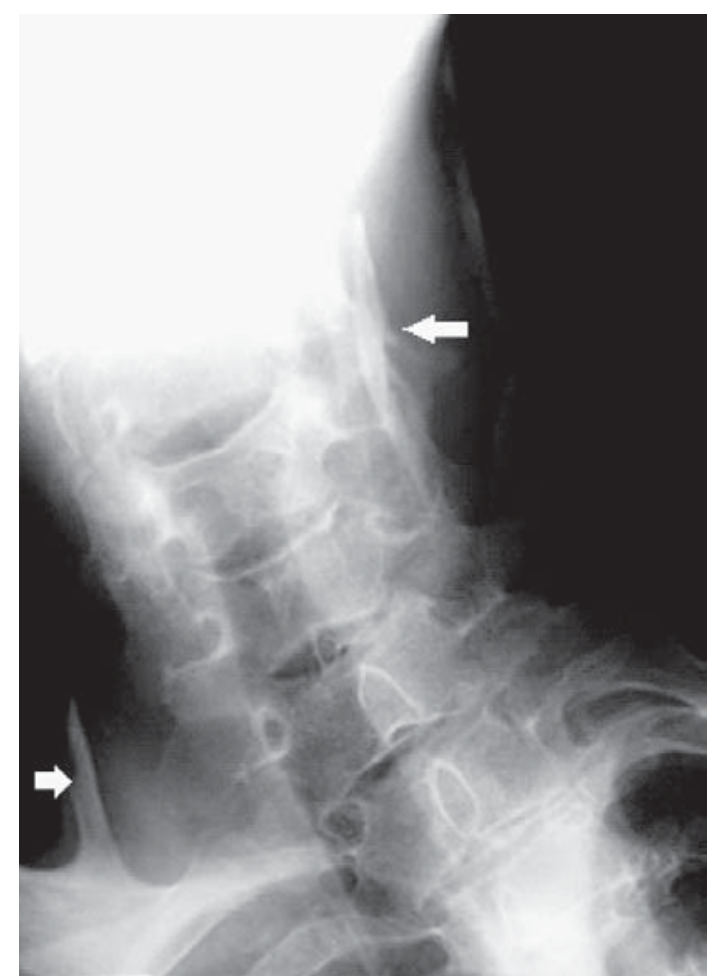

Fig 3. X-ray cervical spine AP view showing heterotropic bone formation in paravertebral and subcutaneous structures (white arrow). Degenerative changes of cervical spine is also evident.

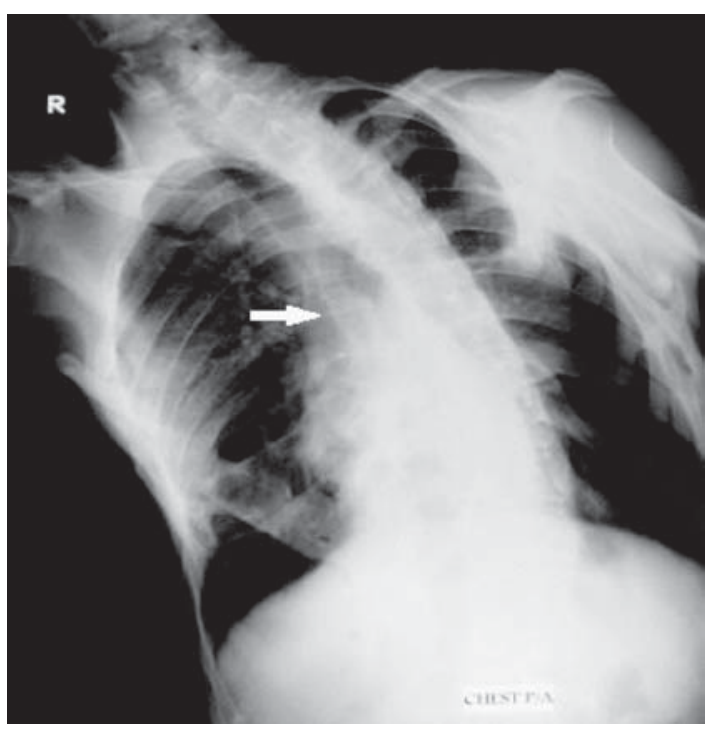

Fig 4. X-ray dorsal spine AP view showing scoliosis with convexity towards left. Orthotopic fusion of dorsal vertebra is seen. Pseudoexostoses with soft tissue ossification is evident (white arrow).

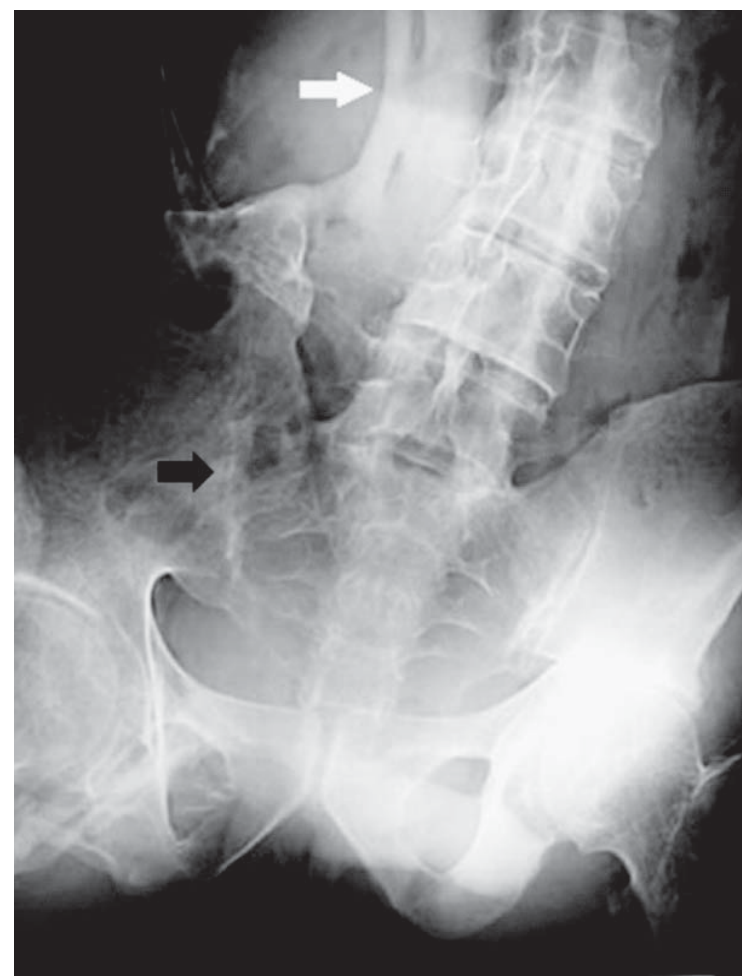

Fig 5. X-ray pelvis AP view showing extensive ligamental and right paravertebral ossification (white arrow). Intervertebral disc calcification is apparent. Right sided osseous bridge is evident between the ilium and sacrum (black arrow).

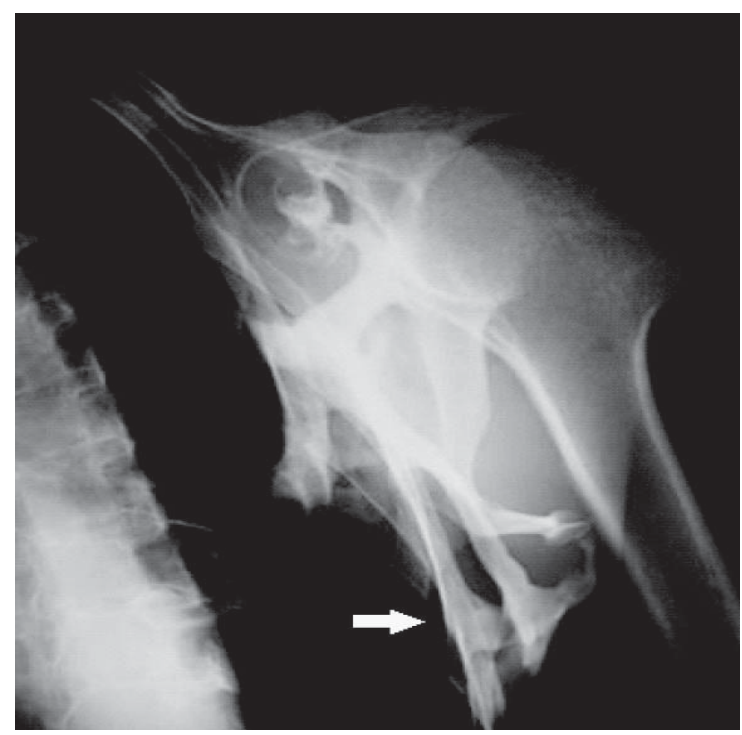

Fig 6. X-ray right shoulder joint oblique view showing diffuse soft tissue ossification overlying the right chest wall and axilla (white arrow) 


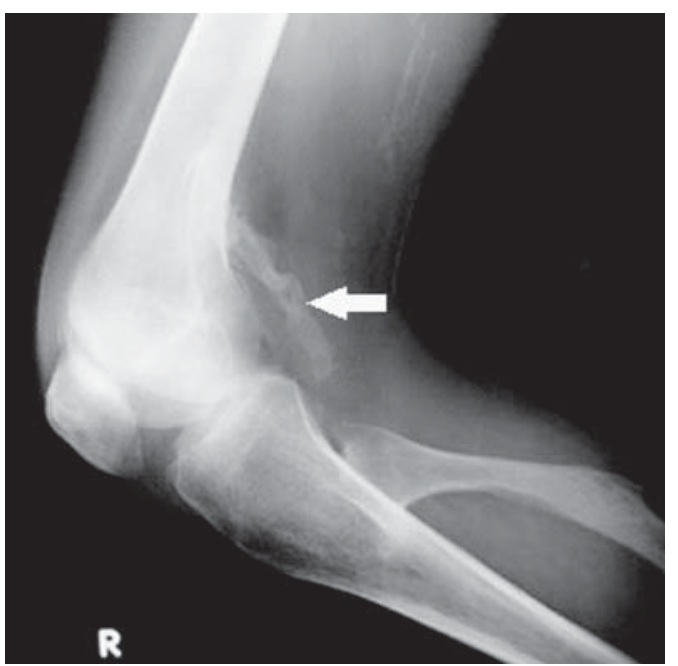

Fig 7. X-ray right knee joint lateral view showing ectopic calcification extending posteriorly from lower end of femur to upper end of tibia (white arrow). Fibula shows pseudoarthrosis in proximal end. Vascular calcification is also evident subcutaneously.

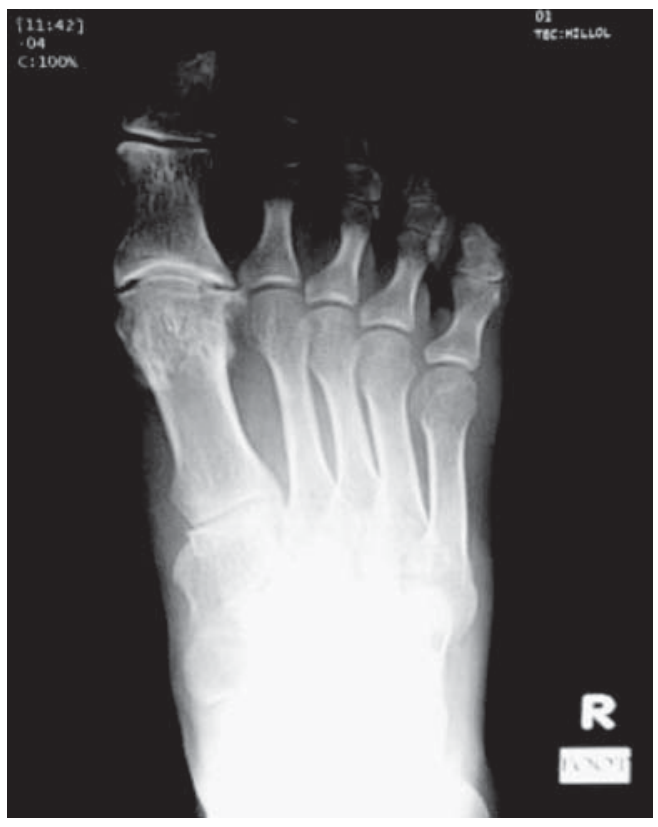

Fig 8. X-ray right foot AP view showing hallux valgus deformity and early degenerative changes of great toe

A 23-year-old male presented in the Orthopedic OPD of Rafatullah Community Hospital (affiliated with TMSS Medical College) with the complaints of rigidity of axial joints and right knee for the last 20 years. He was reasonably well up to 3 years of his age. Then an abscess was noticed over his right shoulder. The village doctor administered several injections of unknown formulations to heal the abscess. The abscess eventually healed, but movement of his neck gradually became restricted. $\mathrm{He}$ then developed painful swellings on his back (Fig 1) as he grew up. These swellings hardened over a period of few days. Subsequently he developed stiffness of spine followed by stiffness of both shoulders and right knee (Fig 2). On examination, the patient had bony hard swellings in cervico-thoraco-lumbar spine and both knees. Both active and passive movements of the spine were restricted in all directions. There were several hard swellings over his back. All other systemic examinations including hearing were normal. Complete blood count showed hypochromic, microcytic anemia. Serum calcium level, alkaline phosphatase and other biochemical parameters were normal. Ossification of muscles was noted in cervical, thoracic and lumbar regions (Figs 3, 4 and 5). There was also ossification around knees and shoulders (Figs 6 and 7). Radiography of both feet showed great toes with hallux valgus deformity (Fig 8).

Patient was advised to take bisphosphonates and NSAIDs. The patient and his family members were counseled of the disease process and its prognosis and were warned that the patient should not undergo surgical procedure in attempt to correct the skeletal deformities.

\section{Discussion}

The first case of FOP was described by Guy Patin in 1692, though first detailed description of FOP was given in 1740 by John Freke, an eminent surgeon in England. McKusick noted that the muscles were only secondarily affected and adopted the term fibrodysplasia in 1972.1,3 Most cases of fibrodysplasia ossificans progressiva result from new mutations in the gene. FOP usually occurs in people with no history of the disorder in their family, as in our case also. In a small number of cases, an affected person has inherited the mutation from one affected parent. ${ }^{1}$ The ACVR1 gene provides instructions for producing a member of a protein family called bone morphogenetic protein type I receptors. It helps to control the growth and development of the bones and muscles, including the gradual replacement of cartilage by bone that occurs in normal skeletal maturation from birth to young adulthood. Researchers believe that a mutation in the ACVR1 gene may change the shape of 
the receptor under certain conditions and disrupts mechanisms that control the receptor's activity. ${ }^{1}$ As a result, the receptor may be constantly turned on. This activation of the receptor causes overgrowth of bone and cartilage and fusion of joints, resulting in the signs and symptoms of FOP. This is known as progressive heterotopic ossification $(\mathrm{HO})$ and it usually begins in the first decade of life. ${ }^{1}$ One important characteristic of FOP is malformation of great toe (shortening or crooking or absence of phalanges). In patient with FOP any injury and viral illnesses often precipitate new flare-ups leading to severe exacerbations of the disorder, resulting in ossification and joint ankylosis. ${ }^{3,4} \mathrm{HO}$ progresses in characteristic anatomic and temporal patterns. Dorsal, axial, cranial, and proximal areas of the body tend to be involved first with ventral, appendicular, caudal, and distal areas involved later. ${ }^{3}$ In this case, it started in the cervical region followed by thoracic and lumbar spine. HO eventually leads to ankylosis of all major joints of the axial and appendicular skeleton restricting movement. ${ }^{3}$ During early childhood, patients with FOP form painful fibrous nodules over the neck, back and shoulders. These nodules often develop after a child experiences some sort of trauma. ${ }^{3}$ Episodes also can occur without any warning. ${ }^{3}$ In most cases, the nodules transform into bone. When the body starts to generate new bone, the patient usually experiences a painful flare-up. Tissue swelling, low grade fever and joint stiffness can occur. Flare-ups can last as long as 6-8 weeks. Bony lesions in FOP have macroscopic features of modeling and remodeling similar to those found in the normotopic skeleton. People who have FOP experience different rates of new bone formation. In some, the progress is rapid, while in others it is more gradual. In some patients hearing impairment may be present. ${ }^{1,3}$

Due to rarity of this disease, the rate of misdiagnosis of the FOP is estimated at $80 \%$ or higher which may lead to unnecessary invasive procedures such as biopsies. ${ }^{3}$ Most common differential diagnoses are cancer, aggressive juvenile fibromatosis, also called desmoid tumors and progressive osseous heteroplasia.

Despite advances in understanding the pathophysiology of FOP, there are no therapies with scientifically proven benefits for the prevention or treatment of FOP. Current approaches to FOP treatment are palliative and modification of symptoms. ${ }^{4,5}$ Surgery is not an option for removing the excess bones because surgery often results in more bone formation. Prevention of soft-tissue injury and protection against the influenza virus remain a hallmark of FOP management because both softtissue injuries and influenza infections can provoke flare-ups of FOP. ${ }^{5}$ Intramuscular injections can also provoke flare-ups and must be avoided. A brief course of high-dose corticosteroids within the first $24 \mathrm{hrs}$ of a flare-up may help to reduce the intense lymphocytic infiltration and tissue edema seen in the early stages of the disease. ${ }^{5}$ Mast cells, lymphocytes and their associated inflammatory mediators may also be reduced with the use of mast-cell stabilizers, leukotriene inhibitors, nonsteroidal anti-inflammatory drugs (NSAIDs). ${ }^{5}$ Angiogenesis, a prominent histopathological feature of preosseous FOP lesions, thus becomes a potential target for therapy. Angiogenesis may potentially be minimized with anti-angiogenic agents, such as NSAIDs (COX-2 inhibitors), thalidomide etc. Etidronate has been studied in FOP because of its inhibitory effect on bone mineralization and its potential to impair ossification at high dosages. ${ }^{4,5}$ The newer aminobisphosphonates do not possess this activity. ${ }^{5}$ Reports have suggested that pamidronate may have beneficial effects either alone or with steroids for the treatment of acute flare-ups. ${ }^{5}$ FOP is a genetic disease and the ultimate treatment may involve a gene correction or a gene bypass approach in the cells and tissues involved in the disease process in future.

\section{References}

1. Kaplan FS. Fibrodysplasia ossificans progressiva-a historical perspective. Clinical Reviews in Bone and Mineral Metabolism 2005; 3(3-4): 179-181.

2. FOP fact sheet. Available at: http://www.ifopa.org/fop-factsheet.html. Accessed April 2014.

3. Mayur K, Sidhartha N, Nagaraja HS. Fibrodysplasia ossificans progressiva in a four year old child. Journal of Orthopaedic Case Reports 2012; 2(2): 17-20.

4. Gosai MM, Hariyani HB. Case report - fibrodysplasia ossificans progressiva. National Journal of Medical Research 2013; 3(1): 100-102.

5. Kaplan FS, Shore EM, Pignolo RJ (eds). The medical management of fibrodysplasia ossificans progressiva: current treatment consideration. The International Clinical Consortium on Fibrodysplasia Ossificans Progressiva, May 2011. Clin Proc Intl Clin Consort FOP 2011; 4: 1-100. 